\title{
COMPARACIÓN DE LAS RESPUESTAS CARDIOVASCULARES AL EJERCICIO Y LA RECUPERACIÓN.
}

\section{COMPARISON OF CARDIOVASCULAR RESPONSES TO EXERCISE AND RECOVERY.}

Autores: ${ }^{1}$ Ricardo Manuel Ortega Oyarvide, ${ }^{2}$ Antonio Ricardo Rodríguez Vargas, ${ }^{3} J u a n$ Arturo Carrión Zalamea, y ${ }^{4}$ Juan Enrique Ramírez Quinteros.

1ORCID ID: https://orcid.org/0000-0003-2692-5792

2ORCID ID: https://orcid.org/0000-0002-4263-6686

3ORCID ID: https://orcid.org/0000-0002-2977-0573

2E-mail de contacto: chico4vb@hotmail.com

Articulo recibido: 28 de Diciembre del 2019

Articulo revisado: 2 de Enero del 2020

Articulo aprobado: 17 de Febrero del 2020

${ }^{1}$ Doctor en Medicina y Cirugía egresado de la Universidad de Guayaquil (Ecuador) con 18 años de experiencia laboral. Posee una maestría en Diseño Curricular de la Universidad de Guayaquil.

${ }^{2}$ Licenciado en Cultura Física egresado del Instituto Superior De Cultura Física Manuel Fajardo (Cuba) con 13 años de experiencia en la docencia. Posee un PhD en Ciencias de la Cultura Física de la Universidad de las Ciencias de la Cultura Física y el Deporte Manuel Fajardo (Cuba).

${ }^{3}$ Medico egresado de la Universidad de Guayaquil (Ecuador) con 2 años de experiencias en la docencia. Posee una especialización en Medicina del Deporte obtenida en la Universidad de Guayaquil (Ecuador).

${ }^{4}$ Doctor en Medicina y Cirugía egresado de la Universidad de Guayaquil (Ecuador) con 18 años de experiencia laboral. Posee una especialización en Medicina del Deporte obtenida en la Universidad de Guayaquil (Ecuador).

\section{Resumen}

El estudio se realizó en 149 deportistas de la Federación Deportiva del Guayas (FedeGuayas) que se preparaban para las diferentes competencias durante sus entrenamientos en las instalaciones de la institución. A los sujetos de investigación se le administraron cargas de trabajo progresivas a partir de $50 \mathrm{~W}$ a $60 \mathrm{rpm}$ en un ergómetro de bicicleta controlado eléctricamente y se incrementaron en pasos de $25 \mathrm{~W}$ por minuto hasta el agotamiento del sujeto. La presión arterial y la frecuencia cardíaca por minuto se registraron durante el ejercicio y la recuperación en intervalos de un minuto durante el transcurso del ejercicio y 15 minutos de recuperación. Sobre la base del estudio, se concluye que las diferencias significativas se demuestran por diferentes categorías de jugadores y estudiantes de educación Física en su presión arterial y respuestas de la frecuencia cardíaca a diferentes intensidades de ejercicio y en diferentes puntos de recuperación.

Palabras claves: Ejercicio máximo, Recuperación, Presión arterial, Frecuencia cardíaca.

\section{Abstract}

The study was conducted in 149 athletes from the Guayas Sports Federation (FedeGuayas) who were preparing for the different competitions during their training at the institution's facilities. The research subjects were given progressive workloads from $50 \mathrm{~W}$ at $60 \mathrm{rpm}$ in an electrically controlled bicycle ergometer and increased in steps of $25 \mathrm{~W}$ per minute until the subject was depleted. Blood pressure and heart rate per minute were recorded during exercise and recovery at intervals of one minute during the course of the exercise and 15 minutes of recovery. Based on the study, it is concluded that significant differences are demonstrated by different categories of physical education players and students in their blood pressure and heart rate responses at different intensities of exercise and at different recovery points.

Keywords: Maximum exercise, Recovery, Blood pressure, Heart rate.

\section{Sumário}

O estudo foi realizado em 149 atletas da Federação de Esportes de Guayas (FedeGuayas) que estavam se preparando para as diferentes competições durante $o$ treinamento nas 
instalações da instituição. Os sujeitos da pesquisa receberam cargas de trabalho progressivas de $50 \mathrm{~W}$ a $60 \mathrm{rpm}$ em um ergômetro de bicicleta controlado eletricamente e aumentaram em etapas de $25 \mathrm{~W}$ por minuto até que o sujeito se esgote. A pressão arterial e a freqüência cardíaca por minuto foram registradas durante o exercício e a recuperação em intervalos de um minuto durante o curso do exercício e 15 minutos de recuperação. Com base no estudo, concluise que diferenças significativas são demonstradas por diferentes categorias de jogadores e estudantes de educação física nas respostas de pressão arterial e frequência cardíaca em diferentes intensidades de exercício e em diferentes pontos de recuperação.

\section{Palavras-chave: Máximo exercício, Recuperação, Pressão sanguínea, Frequência cardíaca.}

\section{Introducción}

Después del ejercicio, los procesos corporales no vuelven inmediatamente al nivel de reposo. La variación en la recuperación del ejercicio ligero, moderado y extenuante está determinada por procesos metabólicos y fisiológicos específicos que resultan de cada nivel de esfuerzo (Hultman et al 1967, Di parampero et al 1983, Gaesser y Brooks, 1984). Durante los minutos iniciales de recuperación, a pesar de que el músculo ya no está trabajando activamente, las demandas de oxígeno no disminuyen inmediatamente; en cambio, el consumo de oxígeno permanece elevado, temporalmente. Esto se debe a la restauración de los procesos metabólicos a sus niveles previos al ejercicio. Este consumo, que excede el generalmente requerido cuando está en reposo, ha sido tradicionalmente referido como la deuda de oxígeno.

Un término más común en la actualidad es el exceso de consumo de oxígeno después del ejercicio (EPOC) (Gaesser y Brooks, 1984). La curva EPOC tiene dos componentes distintos: un componente rápido inicial y un componente secundario lento ambos componentes de la curva reflejan la actividad anaeróbica que se había producido durante el ejercicio. La primera fase de recuperación está marcada por la disminución rápida de $\mathrm{CO}^{2}$ y la frecuencia cardíaca. Es durante este período que las reservas de tejido del ATP y la Fosfocreatina agotadas en el músculo se restauran en 30 segundos y se restauran al $100 \%$ en 3 a 5 minutos (Hultman et al, 1967).

La capacidad de mantener el rendimiento muscular durante el ejercicio de alta intensidad depende del rendimiento de recuperación en muchos deportes individuales y de equipo. Según Zafeiridis (2005), una mayor tasa de resíntesis de la Fosfocreatina, una mayor capacidad para tolerar el tampón y eliminar rápidamente restableciendo el $\mathrm{pH}$ muscular y una mayor actividad de la enzima oxidativa pueden acelerar el proceso de recuperación.

Muchos deportes como el fútbol, el boxeo, hockey, lucha, etc. Tienen un intenso período de trabajo alternando con períodos de descanso cortos a moderados (o reducidos de actividad), dependiendo de las demandas específicas del deporte. No solo es el fosfato de creatina, que es la reserva más inmediata en el músculo esquelético o la resíntesis de ATP, gravado al máximo durante el ejercicio máximo de alta intensidad a corto plazo, el hecho de que las concentraciones de lactato en sangre y músculo también se elevan significativamente después de 6 segundos (Boobis et al. 1983) y 10 segundos (Jacob et al. 1983), siguiendo el trabajo de máxima intensidad, ejemplifica que la glucólisis también presenta una fuente importante de resíntesis de ATP en el ejercicio de corta duración. La caída resultante en el $\mathrm{pH}$ muscular puede tener un efecto perjudicial en el rendimiento posterior (Curtin y Edman, 1989). El regreso de ejercitar el músculo hacia el $\mathrm{pH}$ en reposo y los niveles de $\mathrm{CP}$ normales será, por consiguiente, un componente importante de la recuperación. La alta correlación entre la recuperación durante el ejercicio intermitente y la resíntesis de fosfato de creatina son consistentes con esta teoría (Bogdanis et al, 1996).

Varios estudios han concluido que la recuperación se verá facilitada por una mayor 
capacidad de absorción de oxígeno (Petersen y Cooke, 1994). Por lo tanto, la capacidad de recuperarse rápidamente es importante en muchos deportes de equipo como el fútbol, el hockey y en deportes de combate como el boxeo y la lucha libre. Existen diferencias individuales entre los deportistas para metabolizar el lactato, por ejemplo, la mejora de la aptitud aeróbica desempeña un gran papel en la recuperación. Algunos estudios han respaldado una asociación entre la aptitud aeróbica y la eliminación de lactato (Tomlin y Wenger, 2 (101) después del ejercicio de alta intensidad, mientras que otros no han confirmado una asociación (Evans y Cureton, 1983; Oothuyse y Carter, 1999).

El presente estudio se realizó con el objetivo de comparar los patrones de ejercicio y recuperación con variables cardiovasculares comunes, como la frecuencia cardíaca y la presión arterial, después del ejercicio máximo entre diferentes categorías de deportistas y estudiantes de educación Física.

\section{Desarrollo}

\section{Material y método}

El estudio se realizó en 149 deportistas de la Federación Deportiva del Guayas (FedeGuayas) que se preparaban para las diferentes competencias durante sus entrenamientos en las instalaciones de la institución. El rango de edad de los sujetos fue de 18-25 años. Características medias de edad, altura y peso de los tres grupos se presentan en la tabla 1.

Tabla 1: Comparación de los valores medios de la edad, altura y de peso entre las diferentes categorías de los deportistas.

\begin{tabular}{|l|c|c|c|c|c|c|c|}
\hline Deportes & \multirow{2}{*}{$\begin{array}{c}\text { Número } \\
\text { de }\end{array}$} & \multicolumn{2}{|c|}{$\begin{array}{c}\text { Edad } \\
\text { (Años) }\end{array}$} & \multicolumn{2}{|c|}{$\begin{array}{c}\text { Altura } \\
(\mathrm{cm})\end{array}$} & \multicolumn{2}{|c|}{ Peso (Kg) } \\
\cline { 3 - 8 } & atletas & $\mu$ & $\sigma$ & $\mu$ & $\sigma$ & $\mu$ & $\sigma$ \\
\hline Baloncesto & 29 & 20.52 & 0.78 & 1.78 & 0.08 & 35.86 & 5.01 \\
\hline Balonmano & 11 & 20 & 1 & 1.75 & 0.09 & 30.91 & 3.02 \\
\hline Boxeo & 25 & 22.24 & 2.03 & 1.69 & 0.06 & 31.20 & 3.62 \\
\hline Ciclismo & 21 & 20.33 & 1.98 & 1.75 & 0.05 & 32.14 & 4.05 \\
\hline Fútbol & 52 & 19.17 & 1.44 & 1.73 & 0.06 & 30.67 & 2.63 \\
\hline Voleibol & 11 & 22.36 & 2.25 & 1.76 & 0.08 & 40.91 & 5.39 \\
\hline
\end{tabular}

Se midieron los parámetros cardiovasculares en todos los sujetos en reposo, durante diferentes cargas de trabajo progresivas y diferentes etapas de recuperación.

- Frecuencia cardíaca en latidos / min. Utilizando un monitor de frecuencia cardíaca marca Polar

> Componentes sistólicos y diastólicos de la presión arterial mediante el método auscultatorio esfigmomanómetro y estetoscopio de mercurio

Todos los sujetos recibieron cargas de trabajo progresivas en un ergómetro de bicicleta controlado eléctricamente a partir de $50 \mathrm{~W}$ y luego se aumentó la carga en pasos de $25 \mathrm{~W}$ cada minuto hasta el agotamiento del sujeto. Se le pidió a cada sujeto que mantuviera la frecuencia de pedaleo a $60 \mathrm{rpm}$. Después del agotamiento, se obtuvieron parámetros en términos del ritmo cardiaco y la presión arterial, registrándose a intervalos de un minuto durante un período total de 15 minutos después del ejercicio máximo. Compilando información solamente de los resultados obtenidos según las cargas de trabajo seleccionadas para la realización de esta investigación las cuales fueron:

Frecuencia cardíaca y presión arterial en reposo

> Frecuencia cardíaca y respuesta de la presión arterial a cargas de trabajo de $50 \mathrm{~W}, 100 \mathrm{~W}$ y $150 \mathrm{~W}$

$>$ Ritmo cardíaco y respuesta de la presión arterial en el minuto 3,9 y 15 en la recuperación luego del ejercicio máximo.

\section{Resultados y discusión}

\section{Frecuencia cardíaca y presión arterial en reposo}

La frecuencia cardíaca promedio en reposo varió entre 65,72 a 81,28 latidos / minuto entre las diferentes categorías de jugadores (tabla 2). Se han observado los valores medios más bajos de frecuencia cardíaca en reposo, en el caso de los boxeadores. Estadísticamente hablando, hay una diferencia significativa en los valores de la 
frecuencia cardíaca en reposo entre las diferentes categorías de deportistas y estudiantes de educación Física, como se indica en la tabla 4.

El valor medio de la presión sistólica y diastólica en reposo entre las diferentes categorías de deportistas y los estudiantes de educación Física también revela diferencias significativas evaluadas por análisis de varianza (tablas 4 y 5). Se observa que los ciclistas en lo que respecta a la frecuencia cardiaca, se observa que los boxeadores poseen un valor inferior en comparación con los ciclistas.

Tabla 2: Comparación de la tasa media de frecuencia cardiaca. La presión arterial sistólica y la presión arterial diastólica en reposo entre las diferentes categorías de deportistas y estudiantes de Educación Física

\begin{tabular}{|l|c|c|c|c|c|c|c|}
\hline Deportes & $\begin{array}{c}\text { Número } \\
\text { de } \\
\text { atletas }\end{array}$ & \multicolumn{2}{|c|}{$\begin{array}{c}\text { Frecuencia } \\
\text { cardiaca } \\
\text { (latido/minuto) }\end{array}$} & \multicolumn{2}{|c|}{$\begin{array}{c}\text { Presión } \\
\text { arterial } \\
\text { sistólica } \\
\text { (mm Hg) }\end{array}$} & \multicolumn{2}{|c|}{$\begin{array}{l}\text { Presión } \\
\text { arterial } \\
\text { diastólica } \\
\text { (mm Hg) }\end{array}$} \\
\cline { 2 - 9 } & & $\mu$ & $\sigma$ & $\mu$ & $\sigma$ & $\mu$ & $\sigma$ \\
\hline Baloncesto & 29 & 81.28 & 5.55 & 113.10 & 4.71 & 73.10 & 4.71 \\
\hline Balonmano & 11 & 74.18 & 5.10 & 115.36 & 5.55 & 73.64 & 5.05 \\
\hline Boxeo & 25 & 65.72 & 5.86 & 111.20 & 3.32 & 71.20 & 3.32 \\
\hline Ciclismo & 21 & 76.71 & 7.12 & 118.10 & 6.80 & 76.67 & 4.83 \\
\hline Fútbol & 52 & 70.73 & 6.97 & 113.37 & 4.92 & 73.27 & 4.74 \\
\hline Voleibol & 11 & 75.27 & 4.17 & 115.45 & 5.22 & 75.45 & 5.22 \\
\hline
\end{tabular}

Tabla 3: Comparación de los valores medios de la frecuencia cardiaca en reposo entre las diferentes categorías de deportistas y estudiantes de Educación Física

\begin{tabular}{|c|c|c|c|c|c|}
\hline & Balonmano & Boxeo & Ciclism & Fútbol & Voleibol \\
\hline Baloncesto & \multirow[t]{5}{*}{7.09} & \multirow{2}{*}{\begin{tabular}{c|}
15.56 \\
8.46
\end{tabular}} & \multirow{3}{*}{$\begin{array}{c}4.56 \\
-2.53 \\
-10.99\end{array}$} & \multirow{2}{*}{$\begin{array}{c}10.55 \\
3.45\end{array}$} & \multirow{2}{*}{$\begin{array}{r}6.00 \\
-1.09\end{array}$} \\
\hline Balonmano & & & & & \\
\hline Boxeo & & & & -5.01 & -9.55 \\
\hline Ciclismo & & & & 5.98 & 1.44 \\
\hline Fútbol & & & & & -4.54 \\
\hline
\end{tabular}

Tabla 4: Comparación de los valores medios de la Presión arterial sistólica en reposo entre las diferentes categorías de deportistas y estudiantes de Educación Física.

\begin{tabular}{|c|c|c|c|c|c|}
\hline & Balonman & Boxec & Ciclism & Fútbo & Voleibol \\
\hline Baloncesto & \multirow[t]{5}{*}{-2.26} & 1.90 & -4.99 & -0.26 & -2.35 \\
\hline Balonmano & & \multirow[t]{4}{*}{4.16} & -2.73 & 2.00 & -0.09 \\
\hline Boxeo & & & \multirow[t]{3}{*}{-6.90} & -2.17 & -4.25 \\
\hline Ciclismo & & & & 4.73 & 2.64 \\
\hline Fútbol & & & & & -2.09 \\
\hline
\end{tabular}

Tabla 5: Comparación de los valores medios de la Presión arterial diastólica en reposo entre las diferentes categorías de deportistas y estudiantes de Educación Física.

\begin{tabular}{|l|c|c|c|c|c|}
\hline & Balonmano & Boxeo & Ciclism o & Fútbol & Voleibol \\
\cline { 1 - 1 } Baloncesto & -0.53 & 1.90 & -3.56 & -0.17 & -2.35 \\
\cline { 1 - 1 } Balonmano & & 2.44 & -3.03 & 0.37 & -1.82 \\
\cline { 1 - 4 } Boxeo & & & -5.47 & -2.07 & -425 \\
\cline { 1 - 4 } Ciclismo & & & & 3.40 & 1.21 \\
\cline { 1 - 1 } Fútbol & & & & & -2.19 \\
\hline
\end{tabular}

\section{Respuesta de del ritmo cardiaco y la presión sanguínea al ejercicio.}

Se observa que la administración de ejercicio gradual revela la frecuencia cardíaca de ejercicio diferencial y las respuestas de la presión arterial entre las diferentes categorías de jugadores. Los boxeadores demuestran una frecuencia cardíaca y una respuesta de la presión arterial sistólica significativamente más bajas que todas las demás categorías de jugadores con una carga de trabajo de $50 \mathrm{~W}$. Entre todas las demás categorías de jugadores, las respuestas de la frecuencia cardíaca a $50 \mathrm{~W}$ de carga de trabajo no son estadísticamente diferentes (tablas 6-8).

La respuesta promedio de la Presión arterial sistólica en esta carga de trabajo se ha observado que es significativamente más baja en los boxeadores que en los ciclistas, mientras que no se observa una diferencia estadística en la respuesta de la Presión arterial diastólica entre otras categorías de jugadores. El grupo de voleibolistas también demostró una respuesta significativamente menor en esta variable en comparación con los ciclistas. El componente diastólico de la presión arterial en promedio registró una disminución a la carga de trabajo de $50 \mathrm{~W}$ en comparación con el valor de reposo promedio registrado en las diversas categorías. En términos estadísticos, se observa que los valores de la presión arterial diastólica son comparables entre las diferentes categorías (tabla 67 y 10). 
Tabla 6: Comparación de la tasa media de frecuencia cardiaca. La presión arterial sistólica y la presión arterial diastólica con una carga de trabajo de $50 \mathrm{~W}$ entre las diferentes categorías de deportistas y estudiantes de Educación Física

\begin{tabular}{|l|c|c|c|c|c|c|c|}
\hline Deportes & $\begin{array}{c}\text { Número } \\
\text { de } \\
\text { atletas }\end{array}$ & \multicolumn{2}{|c|}{$\begin{array}{c}\text { Frecuencia } \\
\text { cardiaca } \\
\text { (latido/minuto) }\end{array}$} & \multicolumn{2}{|c|}{$\begin{array}{c}\text { Presión } \\
\text { arterial } \\
\text { sistólica } \\
(\mathrm{mm} \mathrm{Hg})\end{array}$} & \multicolumn{2}{|c|}{$\begin{array}{l}\text { Presión } \\
\text { arterial } \\
\text { diastólica } \\
\text { (mm Hg) }\end{array}$} \\
\cline { 3 - 9 } & & $\mu$ & $\sigma$ & $\mu$ & $\sigma$ & $\mu$ & $\sigma$ \\
\hline Baloncesto & 29 & 147.69 & 7.78 & 149.31 & 7.99 & 59.93 & 3.87 \\
\hline Balonmano & 11 & 146.27 & 8.76 & 150.91 & 7.01 & 60.36 & 1.96 \\
\hline Boxeo & 25 & 135.08 & 7.34 & 146.00 & 6.46 & 58.96 & 2.80 \\
\hline Ciclismo & 21 & 144.33 & 4.65 & 155.24 & 9.81 & 59.48 & 4.31 \\
\hline Fútbol & 52 & 145.13 & 9.09 & 150.00 & 7.92 & 58.56 & 3.21 \\
\hline Voleibol & 11 & 146.27 & 4.43 & 143.64 & 5.05 & 60.55 & 3.70 \\
\hline
\end{tabular}

Tabla 7: Comparación de los valores medios de la frecuencia cardiaca con una carga de trabajo de $50 \mathrm{~W}$ entre las diferentes categorías de deportistas y estudiantes de Educación Física

\begin{tabular}{|l|c|c|c|c|c|}
\hline & Balonmano & Boxeo & Ciclismo & Fútbo & Voleibol \\
\cline { 1 - 1 } Baloncesto & 1.42 & 12.61 & 3.36 & 2.56 & 1.42 \\
\cline { 1 - 1 } Balonmano & & 11.19 & 1.94 & 1.14 & 0 \\
\cline { 1 - 1 } Boxe0 & & & -9.25 & -10.05 & -11.19 \\
\cline { 1 - 1 } Ciclismo & & & & -0.80 & -1.94 \\
\cline { 1 - 1 } & & & & & -1.14 \\
\hline
\end{tabular}

Tabla 8: Comparación de los valores medios de la Presión arterial sistólica con una carga de trabajo de 50W entre las diferentes categorías de deportistas y estudiantes de Educación Física.

\begin{tabular}{|c|c|c|c|c|c|}
\hline & Balonmano & Boxe0 & Ciclismo & Fútbol & Voleibol \\
\hline Baloncesto & -1.60 & 3.31 & -5.93 & -0.69 & 5.67 \\
\hline Balonmano & & 4.91 & -4.33 & 0.91 & 7.27 \\
\hline Boxeo & & & -9.24 & -4.00 & 2.36 \\
\hline Ciclismo & & & & 5.24 & 11.60 \\
\hline Fútbol & & & & & 6.36 \\
\hline
\end{tabular}

La reacción cardiovascular para aumentar aún más la intensidad del ejercicio a $100 \mathrm{~W}$ revela una respuesta similar de la frecuencia cardíaca que se observó a la carga de trabajo inicial de 50W. Los boxeadores demostraron un valor significativamente menor que los otros grupos.

La Presión arterial sistólica respondió significativamente menos vigorosamente en el caso de los boxeadores que los otros grupos, mientras que la respuesta Presión arterial diastólica a la carga de trabajo de $100 \mathrm{~W}$ fue comparable en todos los grupos, excepto para los ciclistas, donde se encontró que era significativamente menor.
Tabla 9: Comparación de la tasa media de frecuencia cardiaca. La presión arterial sistólica y la presión arterial diastólica con una carga de trabajo de $100 \mathrm{~W}$ entre las diferentes categorías de deportistas y estudiantes de Educación Física

\begin{tabular}{|l|c|c|c|c|c|c|c|}
\hline Deportes & $\begin{array}{c}\text { Número } \\
\text { de } \\
\text { atletas }\end{array}$ & \multicolumn{2}{|c|}{$\begin{array}{c}\text { Frecuencia } \\
\text { cardiaca } \\
\text { (latido/minuto) }\end{array}$} & $\begin{array}{l}\text { Presión } \\
\text { arterial } \\
\text { sistólica } \\
(\mathrm{mm}\end{array}$ & \multicolumn{2}{|c|}{$\begin{array}{l}\text { Presión } \\
\text { arterial } \\
\text { diastólica } \\
(\mathrm{mm} \mathrm{Hg})\end{array}$} \\
\cline { 3 - 8 } & & $\mu$ & $\sigma$ & $\mu$ & $\sigma$ & $\mu$ & $\sigma$ \\
\hline Baloncesto & 29 & 170.72 & 7.74 & 174.86 & 8.01 & 59.86 \\
\hline Balonmano & 11 & 171.09 & 8.30 & 179.64 & 4.57 & 60.36 \\
\hline Boxeo & 25 & 156.72 & 9.49 & 175.60 & 6.01 & 59.72 \\
\hline Ciclismo & 21 & 166.33 & 5.03 & 183.81 & 12.03 & 51.48 \\
\hline Fútbol & 52 & 168.25 & 8.66 & 178.37 & 6.40 & 54.79 \\
\hline Voleibol & 11 & 170.45 & 6.09 & 164.91 & & 5.49 & 59.27 \\
\hline
\end{tabular}

Tabla 10: Comparación de los valores medios de la frecuencia cardiaca con una carga de trabajo de $100 \mathrm{~W}$ entre las diferentes categorías de deportistas y estudiantes de Educación Física

\begin{tabular}{|l|c|c|c|c|c|}
\hline & Balonmano & Boxeo & Ciclismo & Fútbo & Voleibol \\
\hline Baloncesto & -0.37 & 14.00 & 4.39 & 2.47 & 0.27 \\
\cline { 1 - 1 } Balonmano & & 14.37 & 4.76 & 2.84 & 0.64 \\
\cline { 1 - 1 } Boxe0 & & & -9.61 & -11.53 & -13.73 \\
\cline { 1 - 1 } Ciclismo & & & & -1.92 & -4.12 \\
\cline { 1 - 1 } & & & & & -2.20 \\
\hline
\end{tabular}

Tabla 11: Comparación de los valores medios de la Presión arterial sistólica con una carga de trabajo de 100W entre las diferentes categorías de deportistas y estudiantes de Educación Física.

\begin{tabular}{|l|c|c|c|c|c|}
\hline & Balonmano & Boxeo & Ciclismo & Fútbo & Voleibol \\
\hline Baloncesto & -4.77 & -0.74 & -8.95 & -3.50 & 9.95 \\
\cline { 1 - 1 } Balonmano & & 4.04 & -4.17 & 1.27 & 14.73 \\
\cline { 1 - 1 } Boxeo & & & -8.21 & -2.77 & 10.69 \\
\cline { 1 - 1 } Ciclismo & & & & 5.44 & 18.90 \\
\cline { 1 - 1 } Fútbol & & & & & 13.46 \\
\hline
\end{tabular}

La comparación del ritmo cardíaco por minuto según la respuesta a la carga de trabajo de 150 W entre diferentes grupos estudiados de deportistas revela una menor respuesta en el caso de los boxeadores de manera significativa y los voleibolistas registraron el valor de frecuencia cardíaca que es significativamente más alta que la media del ritmo cardíaco de boxeadores, ciclistas y futbolistas.

La respuesta de presión arterial sistólica a la carga de trabajo $150 \mathrm{~W}$ presento su máxima reacción de los ciclistas seguido de balonmano, fútbol, boxeadores, y voleibol en orden 
decreciente En términos estadísticos, se han registrado diferencias significativas en la respuesta de presión arterial sistólica entre las diferentes categorías.

Tabla 12: Comparación de la tasa media de frecuencia cardiaca. La presión arterial sistólica y la presión arterial diastólica con una carga de trabajo de $150 \mathrm{~W}$ entre las diferentes categorías de deportistas y estudiantes de Educación Física.

\begin{tabular}{|l|c|c|c|c|c|c|c|}
\hline Deportes & $\begin{array}{c}\text { Número } \\
\text { de } \\
\text { atletas }\end{array}$ & \multicolumn{2}{|c|}{$\begin{array}{c}\text { Frecuencia } \\
\text { cardiaca } \\
\text { (latido/minuto) }\end{array}$} & $\begin{array}{l}\text { Presión } \\
\text { arterial } \\
\text { sistólica } \\
(\mathrm{mm} \\
\mathrm{Hg}) \text { ) }\end{array}$ & $\begin{array}{l}\text { Presión } \\
\text { arterial } \\
\text { diastólica } \\
(\mathrm{mm} \mathrm{Hg})\end{array}$ \\
\cline { 3 - 8 } & & $\mu$ & $\sigma$ & $\mu$ & $\sigma$ & $\mu$ & $\sigma$ \\
\hline Baloncesto & 29 & 186.17 & 6.23 & 196.79 & & 5.61 & 60.55 \\
\hline Balonmano & 11 & 184.09 & 6.06 & 201.45 & 5.15 & 60.18 \\
\hline Boxeo & 25 & 170.28 & 12.43 & 198.80 & 4.15 & 58.84 \\
\hline Ciclismo & 21 & 183.10 & 6.07 & 209.81 & 16.60 & 46.19 \\
\hline Fútbol & 52 & 181.29 & 9.41 & 200.98 & 4.72 & 54.00 \\
\hline Voleibol & 11 & 193.18 & 6.15 & 186.55 & 7.63 & 59.81 \\
\hline
\end{tabular}

Tabla 13: Comparación de los valores medios de la frecuencia cardiaca con una carga de trabajo de $150 \mathrm{~W}$ entre las diferentes categorías de deportistas y estudiantes de Educación Física

\begin{tabular}{|c|c|c|c|c|c|}
\hline & \multicolumn{5}{|c|}{ Balonmano|Boxeo|Ciclismo|FútbolVoleibol } \\
\hline Baloncesto & 2.08 & 15.89 & 3.08 & 4.88 & -7.01 \\
\hline Balonmano & & 13.81 & 1.00 & 2.80 & -9.09 \\
\hline Boxeo & & & -12.82 & -11.01 & -22.90 \\
\hline Ciclismo & & & & 1.81 & -10.99 \\
\hline Fútbol & & & & & -11.89 \\
\hline
\end{tabular}

Tabla 14: Comparación de los valores medios de la Presión arterial sistólica con una carga de trabajo de 150W entre las diferentes categorías de deportistas y estudiantes de Educación Física.

\begin{tabular}{|c|c|c|c|c|c|}
\hline & Balonman & Boxeo & Ciclism & Fútbol & Voleibol \\
\hline Baloncesto & -4.66 & -2.01 & -13.02 & -4.19 & 10.25 \\
\hline Balonmano & & 2.65 & -8.36 & 0.47 & 14.91 \\
\hline Boxeo & & & -11.01 & -2.18 & 12.25 \\
\hline Ciclismo & & & & 8.83 & 23.26 \\
\hline Fútbol & & & & & 14.44 \\
\hline
\end{tabular}

De manera similar, la Presión arterial diastólica también se ha observado que responde a diferentes grados entre los diversos grupos. Con excepción de los ciclistas, boxeadores y futbolistas valores de Presión arterial diastólica son significativamente más bajos que en comparación con los otros grupos.
Tabla 15: Comparación post hoc de Schaeffe para las diferencias de medias en la presión arterial diastólica a una carga de trabajo de $150 \mathrm{~W}$ entre las categorías de deportistas y estudiantes de Educación Física.

\begin{tabular}{|c|c|c|c|c|c|}
\hline & Balonmar & Boxes & Ciclism & Fútbc & Voleibol \\
\hline Baloncesto & \multirow[t]{5}{*}{0.37} & 1.71 & 14.36 & 6.55 & 0.73 \\
\hline Balonmano & & \multirow[t]{4}{*}{1.34} & 13.99 & 6.18 & 0.36 \\
\hline Boxe0 & & & 12.65 & 4.84 & -0.98 \\
\hline Ciclismo & & & & -7.81 & -13.63 \\
\hline Fútbol & & & & & -5.82 \\
\hline
\end{tabular}

\section{Recuperación de la frecuencia cardíaca y la presión arterial después del ejercicio máximo.}

La comparación de la recuperación inicial de la frecuencia cardíaca después del ejercicio exhaustivo, medida en el tercer minuto de recuperación entre los diversos grupos, demuestra el valor medio más bajo de frecuencia cardiaca de 101.81 latidos / min en el caso de ciclistas seguidos de boxeadores (103.44), balonmano (105.73), fútbol (106.08), baloncesto (112.21) y voleibol (118.55) en orden creciente. En otras palabras, se observa que los ciclistas, los boxeadores y el grupo de jugadores de fútbol se recuperan relativamente más rápido en comparación con los otros grupos. Estadísticamente hablando, se observan diferencias significativas en el tercer minuto del período de recuperación en los valores de frecuencia cardíaca por minuto entre los diversos grupos.

Tabla 16: Comparación de los valores de recuperación de frecuencia cardiaca de recuperación de 3 minutos entre las categorías de deportistas y estudiantes de Educación Física.

\begin{tabular}{|l|c|c|c|c|c|c|c|}
\hline Deportes & $\begin{array}{c}\text { Número } \\
\text { de } \\
\text { atletas }\end{array}$ & \multicolumn{2}{|c|}{$\begin{array}{c}\text { Frecuencia } \\
\text { cardiaca } \\
\text { latido/minuto) }\end{array}$} & \multicolumn{2}{|c|}{$\begin{array}{c}\text { Presión } \\
\text { arterial } \\
\text { sistólica } \\
(\mathrm{mm} \mathrm{Hg})\end{array}$} & \multicolumn{2}{|c|}{$\begin{array}{l}\text { Presión } \\
\text { arterial } \\
\text { diastólica } \\
(\mathrm{mm} \mathrm{Hg})\end{array}$} \\
\cline { 3 - 8 } & & $\mu$ & $\sigma$ & $\mu$ & $\sigma$ & $\mu$ & $\sigma$ \\
\hline Baloncesto & 29 & 112.21 & 6.37 & 131.21 & 6.77 & 60.48 & 3.88 \\
\hline Balonmano & 11 & 105.73 & 2.53 & 122.73 & 4.67 & 60.36 & 4.37 \\
\hline Boxeo & 25 & 103.44 & 8.43 & 125.20 & 8.95 & 62.72 & 5.22 \\
\hline Ciclismo & 21 & 101.81 & 8.37 & 126.43 & 7.93 & 63.24 & 5.64 \\
\hline Fútbol & 52 & 106.08 & 5.97 & 126.63 & 7.19 & 51.69 & 5.39 \\
\hline Voleibol & 11 & 118.55 & 7.17 & 136.82 & 7.17 & 59.36 & 3.17 \\
\hline
\end{tabular}


Tabla 17: Comparación post hoc de Scheffe para las diferencias medias en la frecuencia cardíaca de recuperación de 3 minutos entre las categorías de deportistas y estudiantes de Educación Física.

\begin{tabular}{|l|c|c|c|c|c|}
\hline & Balonmano & Boxeo & Ciclismo & Fútbo & Voleibol \\
\hline Baloncesto & 6.48 & 8.77 & 10.40 & 6.13 & -6.34 \\
\cline { 1 - 1 } Balonmano & & 2.29 & 3.92 & -0.35 & -12.82 \\
\cline { 1 - 1 } Boxe0 & & & 1.63 & -2.64 & -15.11 \\
\cline { 1 - 1 } Ciclismo & & & & -4.27 & -16.74 \\
\cline { 1 - 4 } Fútbol & & & & & -12.47 \\
\hline
\end{tabular}

La frecuencia cardíaca de recuperación al tercer minuto de recuperación después del ejercicio máximo revela valores más altos en el caso del grupo de baloncesto y voleibol en comparación con los otros grupos. En términos estadísticos, los ciclistas, boxeadores, jugadores de fútbol y balonmano registran valores frecuencia cardiaca significativamente más bajos que los grupos de baloncesto y voleibol. En otras palabras, la presión sistólica muestra una tendencia a regresar al estado de reposo más rápido en el caso de ciclistas, boxeadores, jugadores de fútbol y balonmano que los grupos de baloncesto y voleibol.

Tabla 18: Comparación post hoc de Scheffe para las diferencias de medias en la presión sistólica de recuperación de 3 minutos entre las categorías de deportistas y estudiantes de Educación Física.

\begin{tabular}{|c|c|c|c|c|c|}
\hline & Balonman & Boxe0 & Ciclismo & Fútbo & Voleibol \\
\hline Baloncesto & 8.48 & 6.01 & 4.78 & 4.57 & -5.61 \\
\hline Balonmano & & -2.47 & -3.70 & -3.91 & -14.09 \\
\hline Boxe0 & & & -1.23 & -1.43 & -11.62 \\
\hline Ciclismo & & & & -0.21 & -10.39 \\
\hline Fútbol & & & & & -10.18 \\
\hline
\end{tabular}

La medición de la Presión arterial diastólica después de tres minutos de interrupción del ejercicio máximo revela valores casi comparables. Estadísticamente hablando, no se han observado diferencias significativas (Tabla 16).

La progresión del período de recuperación a nueve minutos después del ejercicio máximo revela una disminución adicional en los valores promedio de pulsaciones por minutos registrados en los diversos grupos (Tabla 19).

Los ciclistas, boxeadores y jugadores de fútbol exhiben la misma tendencia de recuperación más rápida que sus otras contrapartes, como se observó en el tercer minuto de recuperación. El análisis de varianza revela la existencia de diferencias significativas en la frecuencia cardiaca registradas a los 9 minutos de recuperación. La comparación post hoc de Scheffe revela que los ciclistas, boxeadores y jugadores de fútbol se recuperan significativamente más rápido que el grupo de balonmano.

Tabla 19: Comparación de los valores de frecuencia cardiaca, presión arterial sistólica y presión arterial diastólica de recuperación de 9 minutos entre las categorías de deportistas y estudiantes de Educación Física.

\begin{tabular}{|l|c|c|c|c|c|c|c|}
\hline Deportes & $\begin{array}{c}\text { Número } \\
\text { de } \\
\text { atletas }\end{array}$ & \multicolumn{2}{|c|}{$\begin{array}{c}\text { Frecuencia } \\
\text { cardiaca } \\
\text { latido/minuto) }\end{array}$} & \multicolumn{2}{|c|}{$\begin{array}{c}\text { Presión } \\
\text { arterial } \\
\text { sistólica } \\
(\mathrm{mm} \mathrm{Hg})\end{array}$} & \multicolumn{2}{|c|}{$\begin{array}{l}\text { Presión } \\
\text { arterial } \\
\text { diastólica } \\
(\mathrm{mm} \mathrm{Hg})\end{array}$} \\
\cline { 2 - 9 } & & $\mu$ & $\sigma$ & $\mu$ & $\sigma$ & $\mu$ & $\sigma$ \\
\hline Baloncesto & 29 & 100.97 & 4.90 & 116.21 & 4.75 & 73.79 & 4.94 \\
\hline Balonmano & 11 & 97.27 & 2.10 & 113.64 & 5.05 & 72.73 & 4.67 \\
\hline Boxeo & 25 & 94.52 & 5.45 & 114.40 & 5.07 & 72.00 & 5.00 \\
\hline Ciclismo & 21 & 92.76 & 6.25 & 117.86 & 6.04 & 76.67 & 4.83 \\
\hline Fútbol & 52 & 95.98 & 4.79 & 114.04 & 6.03 & 73.08 & 5.44 \\
\hline Voleibol & 11 & 106.27 & 6.33 & 124.36 & 5.43 & 70.91 & 3.02 \\
\hline
\end{tabular}

Tabla 20: Comparación post hoc de Scheffe para las diferencias de medias en la frecuencia cardiaca durante el periodo de recuperación a los 9 minutos entre las categorías de deportistas y estudiantes de Educación Física.

\begin{tabular}{|l|c|c|c|c|c|}
\hline & Balonmano & Boxeo & Ciclism & Fútbol & Voleibol \\
\hline Baloncesto & 3.69 & 6.45 & 8.20 & 4.98 & -5.31 \\
\cline { 1 - 1 } Balonmano & & 2.75 & 4.51 & 1.29 & -9.00 \\
\cline { 1 - 1 } Boxe0 & & & 1.76 & -1.46 & -11.75 \\
\cline { 1 - 1 } Ciclismo & & & & -3.22 & -13.51 \\
\cline { 1 - 1 } & & & & & -10.29 \\
\hline
\end{tabular}

La Presión arterial sistólica tiende a alcanzar valores promedio casi similares al noveno minuto de recuperación en los diversos grupos, excepto en el caso del grupo de balonmano donde se observa un valor medio relativamente mayor de $124.36 \mathrm{~mm} \mathrm{Hg}$. En términos estadísticos, se encuentra que es significativamente mayor que todos los otros grupos. 
Tabla 21: Comparación post hoc de Scheffe para las diferencias de medias en la presión arterial sistólica durante el periodo de recuperación a los 9 minutos entre las categorías de deportistas y estudiantes de Educación Física.

\begin{tabular}{|c|c|c|c|c|c|}
\hline & \multicolumn{5}{|c|}{ Balonmano|Boxeo|Ciclismo Fútbol|Voleibol } \\
\hline Baloncesto & 2.57 & 1.81 & -1.65 & 2.17 & -8.16 \\
\hline Balonmano & & -0.76 & -4.22 & -0.40 & -10.73 \\
\hline Boxe0 & & & -3.46 & 0.36 & -9.96 \\
\hline Ciclismo & & & & 3.82 & -6 \\
\hline Fútbol & & & & & -10.3 \\
\hline
\end{tabular}

La recuperación de la presión arterial diastólica a los 9 minutos del cese del ejercicio máximo revela valores casi comparables. Estadísticamente hablando, no se han observado diferencias significativas entre los diferentes grupos.

La restauración cardiovascular para un mayor avance en la recuperación hasta el minuto 15 demuestra una disminución continua en la frecuencia cardíaca por minuto, aunque a un ritmo más lento. Es similar a la observada en el tercer y noveno minuto de las fases de recuperación. Se observa que el grupo de balonmano se recupera más lentamente de todos los otros grupos.

Tabla 22: Comparación de los valores de recuperación de 15 minutos de frecuencia cardiaca, presión arterial sistólica y la presión arterial diastólica entre las categorías de deportistas y estudiantes de Educación Física.

\begin{tabular}{|l|c|c|c|c|c|c|c|}
\hline Deportes & $\begin{array}{c}\text { Número } \\
\text { de } \\
\text { atletas }\end{array}$ & \multicolumn{2}{|c|}{$\begin{array}{c}\text { Frecuencia } \\
\text { cardiaca } \\
\text { (latido/minuto) }\end{array}$} & \multicolumn{2}{|c|}{$\begin{array}{l}\text { Presión } \\
\text { arterial } \\
\text { sistólica } \\
(\mathrm{mm} \mathrm{Hg})\end{array}$} & \multicolumn{2}{|c|}{$\begin{array}{l}\text { Presión } \\
\text { arterial } \\
\text { diastólica } \\
(\mathrm{mm} \mathrm{Hg})\end{array}$} \\
\cline { 3 - 8 } & & $\mu$ & $\sigma$ & $\mu$ & $\sigma$ & $\mu$ & $\sigma$ \\
\hline Baloncesto & 29 & 93.93 & 3.91 & 109.31 & 5.30 & 73.45 & 4.84 \\
\hline Balonmano & 11 & 92.55 & 1.75 & 104.55 & 5.22 & 73.64 & 5.05 \\
\hline Boxeo & 25 & 87.44 & 4.90 & 103.60 & 4.68 & 72.40 & 4.36 \\
\hline Ciclismo & 21 & 88.10 & 4.57 & 108.81 & 6.31 & 76.67 & 4.83 \\
\hline Fútbol & 52 & 91.52 & 3.98 & 104.13 & 4.82 & 73.46 & 4.80 \\
\hline Voleibol & 11 & 100.91 & 6.20 & 114.55 & 5.22 & 73.64 & 5.04 \\
\hline
\end{tabular}

Tabla 23: Comparación post hoc de Scheffe para las diferencias de medias en la recuperación de 15 minutos de la frecuencia cardiaca entre las categorías de deportistas y estudiantes de Educación Física.

\begin{tabular}{|c|c|c|c|c|c|}
\hline & \multicolumn{5}{|c|}{ Balonmano|Boxeo Ciclismo|Fútbol Voleibol } \\
\hline Baloncesto & 1.39 & 6.49 & 5.84 & 2.41 & -6.98 \\
\hline Balonmano & & 5.11 & 4.45 & 1.03 & -8.36 \\
\hline Boxe0 & & & -0.66 & -4.08 & -13.47 \\
\hline Ciclismo & & & & -3.42 & -12.81 \\
\hline Fútbol & & & & & -9.39 \\
\hline
\end{tabular}

El componente sistólico de la presión arterial continúa disminuyendo en todos los grupos y se observa que incluso cae por debajo de los niveles de reposo. Sin embargo, las diferencias entre grupos se observan en la presión arterial sistólica y también tienen significación estadística. El grupo de jugadores de balonmano demuestra un valor medio significativamente mayor de presión arterial sistólica que los otros grupos. La presión arterial diastólica por otro lado muestra valores similares y estadísticamente no son diferentes entre sí.

Tabla 24: Comparación post hoc de Scheffe para las diferencias de medias en la recuperación de 15 minutos presión arterial sistólica entre las categorías de deportistas y estudiantes de Educación Física.

\begin{tabular}{|c|c|c|c|c|c|}
\hline & Balonman & Boxed & Ciclism & Fútbo & Voleibol \\
\hline Baloncesto & 1.39 & 6.49 & 5.84 & 2.41 & -6.98 \\
\hline Balonmano & & 5.11 & 4.45 & 1.03 & -8.36 \\
\hline Boxeo & & & -0.66 & -4.08 & -13.47 \\
\hline Ciclismo & & & & -3.42 & -12.81 \\
\hline Fútbol & & & & & -9.39 \\
\hline
\end{tabular}

En la presente investigación también se ha estudiado la respuesta sistólica de la presión arterial en relación con la frecuencia cardiaca durante el ejercicio y la recuperación, se observa a partir de las cifras que la presión arterial aumenta con el aumento de la frecuencia cardiaca durante el ejercicio graduado en todas las categorías de jugadores.

Es interesante observar que la respuesta de presión arterial diastólica al ejercicio en relación con la frecuencia cardiaca es leve hasta la aceleración de 150 latidos / minuto, donde después de la presión arterial sistólica aumenta más vigorosamente. En otras palabras, la dependiente de la respuesta durante el ejercicio que conduce a un aumento de la frecuencia cardíaca de hasta 150 latidos / minuto es menos pronunciada.

\section{Conclusión}

Los boxeadores, ciclistas y jugadores de fútbol exhiben una respuesta presión arterial sistólica más pronunciada después de alcanzar 150 latidos / minuto de frecuencia cardiaca en comparación con otras categorías. La observación sugiere una estimulación simpática 
más intensiva lograda por los boxeadores, ciclistas y futbolistas más allá de 150 latidos / min de ejercicio. Esta estimulación les ayuda a lograr un mayor gasto cardíaco y, por lo tanto, aumenta su capacidad para hacer ejercicio. El gasto cardíaco aumenta de forma rectilínea y se estabiliza en el ejercicio máximo. El aumento inicial en el gasto cardíaco refleja un aumento en el volumen sistólico y la frecuencia cardíaca; sin embargo, con una carga de trabajo superior al 40-50\% de volumen máximo, el aumento en el gasto cardíaco se logra únicamente mediante aumentos en la frecuencia cardíaca. En individuos normalmente activos, el volumen sistólico aumenta inicialmente y luego se estabiliza en aproximadamente $40-50 \%$ del volumen máximo (Astrand et al, 1964 y Higginbotham et al, 1986). El volumen sistólico en realidad puede disminuir ligeramente cerca del final del ejercicio máximo en individuos no entrenados y moderadamente entrenados (Gledhill y Jamnik, 1994). El volumen diastólico final del ventrículo izquierdo aumenta en gran medida debido al retorno de la sangre al corazón por la bomba muscular activa y al aumento del flujo de salida simpático hacia las venas que causa vasoconstricción y aumenta el retorno venoso. El volumen del extremo sistólico del ventrículo izquierdo disminuye debido a la contractilidad aumentada del corazón, que expulsa más sangre del ventrículo y deja menos en el ventrículo (Poliner et al, 1980).

La frecuencia cardíaca aumenta en forma rectilínea y mesetas en el ejercicio máximo. La presión arterial sistólica aumenta durante el ejercicio máximo, a menudo alcanzando valores durante el ejercicio de $200 \mathrm{~mm} \mathrm{Hg}$ en individuos muy en forma. El aumento de la presión arterial sistólica es causado por el aumento del gasto cardíaco que supera la disminución de la resistencia. La presión arterial sistólica y la frecuencia cardíaca son dos variables que se controlan de forma rutinaria durante una prueba de ejercicio para garantizar la seguridad de los participantes. Si alguna de estas variables no aumenta con una carga de trabajo cada vez mayor, es posible la insuficiencia cardiovascular.
La caída abrupta de la frecuencia cardíaca durante el primer minuto de recuperación puede explicarse en los hallazgos de muchos investigadores que informaron la retirada de la estimulación simpática intensiva lograda durante el ejercicio (Gaesser y Brooks, 1984 y. Zafeiridis et al, 2005).

Sobre la base del estudio, se concluye que las diferentes categorías de jugadores y estudiantes de educación física demuestran diferencias significativas en sus respuestas de presión arterial y frecuencia cardíaca a diferentes intensidades de ejercicio y en diferentes puntos de recuperación.

\section{Referencias Bibliográficas}

American College of Sport Medicine 1995. Guidance for exercise testing and prescription (5th edition). Philadelphia: Lea and Febiger

Astrand, P.O.; Cuddy; T.E., Saltine, B. and Stenberg, J. 1964. Cardiac out put during sub maximal and maximal work. Journal of Applied Physiology 2(19): 268-274.

Balsom, P.D.; Ekblom, B; Sjodin, C. 1994. Enhanced oxygen availability during high intensity intermittent exercise anaerobic metabolic concentrations in blood. Acta Physiol. Scand. 150: 455-456.

Bogdanis, G.C., Nevill, M.E., Bobbis, L.H., Lakmy, K.A., and Nevill, A.M. 1995. Recovery of power out put and muscle metabolites following 30-s of maximal sprint cycling in man. J. Physiol, 482: 467-480.

Boobis, LH; William, C. and Wooten, S.A. 1983. Human muscle metabolism during brief maximal exercise. J. Physiol., 338: 2122.

Curtin, N.A. and Edman, K.A. 1989. Effect of fatigue and reduced intercellular $\mathrm{pH}$ on segment dynamics in isometric relaxation of frog muscle fibers. J Physiol., 299: 465-484.

Di prampero, P.E., Mahler, P., Giezendarnner, D. and Cerretelli, P. 1983. The effect of priming exercise on $\mathrm{VO} 2$ kinetics and $\mathrm{O} 2$ deficit at the onset of stepping and cycling. J. Appl. Physiol., 66: 2023-2031.

Evans, B.W. \& Cureston, K.J. 1983. Effect of physical conditioning on blood lactate 
disappearance after supramaximal exercise. Br. J. Sports Medicine, 17: 40-45.

Gaesser, G.A. and Brooks, G.A. 1984. Metabolic bases of exercise post-exercise oxygen consumption: A review. Med. Sci. Sports. Exercise, 16: 29-43

Gledhill, N., Cox, D. and Jamnik, R. 1994. Endurance athletes' stroke volume does not plateau: Major advance is diastolic function. Medicine and Science in Sports and Exercise, 26: 1116- 1121.

Higginbotham, M.B., Morries, K.G., Willams, R.S., McHale, P.A., Coleman, R.E. and Cobb, F.R. 1986. Regulation of stroke volume during sub maximal and maximal upright exercise in normal man. Circulation Research 58: 281- 291.

Holloszy, J.O. and Coyle, E.F. 1984. Adaptation of skeletal muscle to endurance exercise and their metabolic consequences. J. Appl. Phsiol., 56: 831-838.

Hultman, E. and Harris, R.C. 1976. The time course of phosphorylcreatin resynthesis during recovery of the quadriceps muscle in man. Pflugers Arch., 367: 137-142.

Jacobs, I., Tesch, P.A., Bar-or, O., Karlsson, J. and Dotal, R. 1983. Lactate in human skeletal muscle after 10second and 30second of supramaximal exercise. J. Appl. Physiol., 55: 365-367.

Karlsson, J. 1971. Lactate and phosphates concentration in working muscles of man with special reference to oxygen deficit at the onset of work. Acta Physiol. Suppl., 358: 7.

Oosthuse, T. \& Carter, R.N. 1999. Plasma lactate decline during passive recovery from high intensity exercise. Med. Sports Exerc., 31: 676-684.
Peter, T. 2005. Aerobic energy and aerobic conditioning- Human Kinetic publishers.Available on line: http://www. Humankinetics.com/ products.

Poliner, L.R. Dehmer, G.J., Lewis, S.E., Parkey R.W., Blomqvist, C.G. and Willerson, J.T. 1980. Left ventricular performance in normal subjects. A comparison of the responses to exercise in the upright supine positions Circulation, 62: 528-534

Singorile, J. F. Ingals, C. 1993. The effect of active and passive recovery on short term high intensity power output. Canadian Journal of Applied Physiology, 18:31-42.

Tomli, D.L. \& Wenger, H.A. 2001. The relationship between aerobic fitness \& recovery from high intensity intermittent exercise. J. Sports Med., 31(1): 1-11.

Yoshida, T., Watari, H., and Tagawa 1996. Effect of active and passive recoveries on splitting of inorganic phosphate peak determined by 31 nuclear magnetic resonance spectroscopies. NMR-Biomed., 9: 13-19.

Zafeiridis, A., Dalamitros, A., Dipla, K., Manou, V., Galanis, N. and Kellis, S. 2005. Recovery during high-intensity intermittent anaerobic exercise in boys, teens and men. J. Medicine and Science in Sport Exercise, 37(3): 505-512.

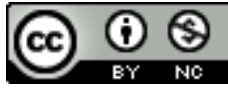
Creative Commons Reconocimiento-No Comercial 4.0 Internacional. Copyright (c) Ricardo Manuel Ortega Oyarvide, Antonio Ricardo Rodríguez Vargas, Juan Arturo Carrión Zalamea, y Juan Enrique Ramírez Quinteros. 